\title{
Selective Hydrogenation of Monosubstituted Alkenes by Pd Nanoparticles Embedded in Polyelectrolyte Films
}

Somnath Bhattacharjee and Merlin Bruening*

Department of Chemistry, Michigan State University, East Lansing, MI 48824

\section{Supporting information}

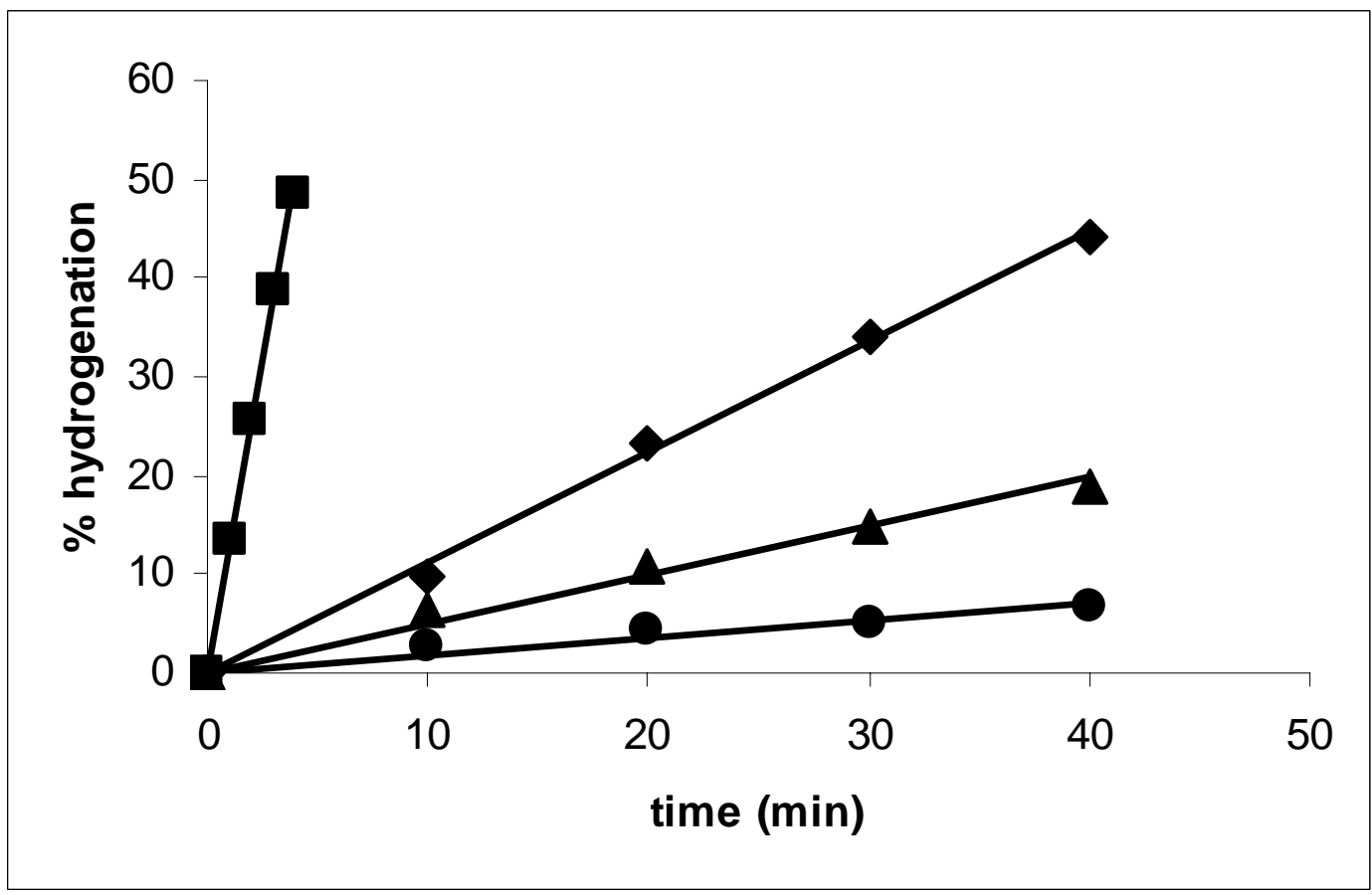

Figure S1. Percent of substrates hydrogenated vs reaction time in water for allyl alcohol (1, squares), 2-methyl-2-propen-1-ol (2, triangles), crotyl alcohol (3, diamonds) and 3methyl-2-buten-1-ol (4, circles). [PAA/PEI-Pd(0)] 3 PAA-coated alumina was used as a catalyst. Percent hydrogenation was calculated as moles product/(moles product + moles reactant + moles isomer). Lines are a fit to the data forced through the origin. 


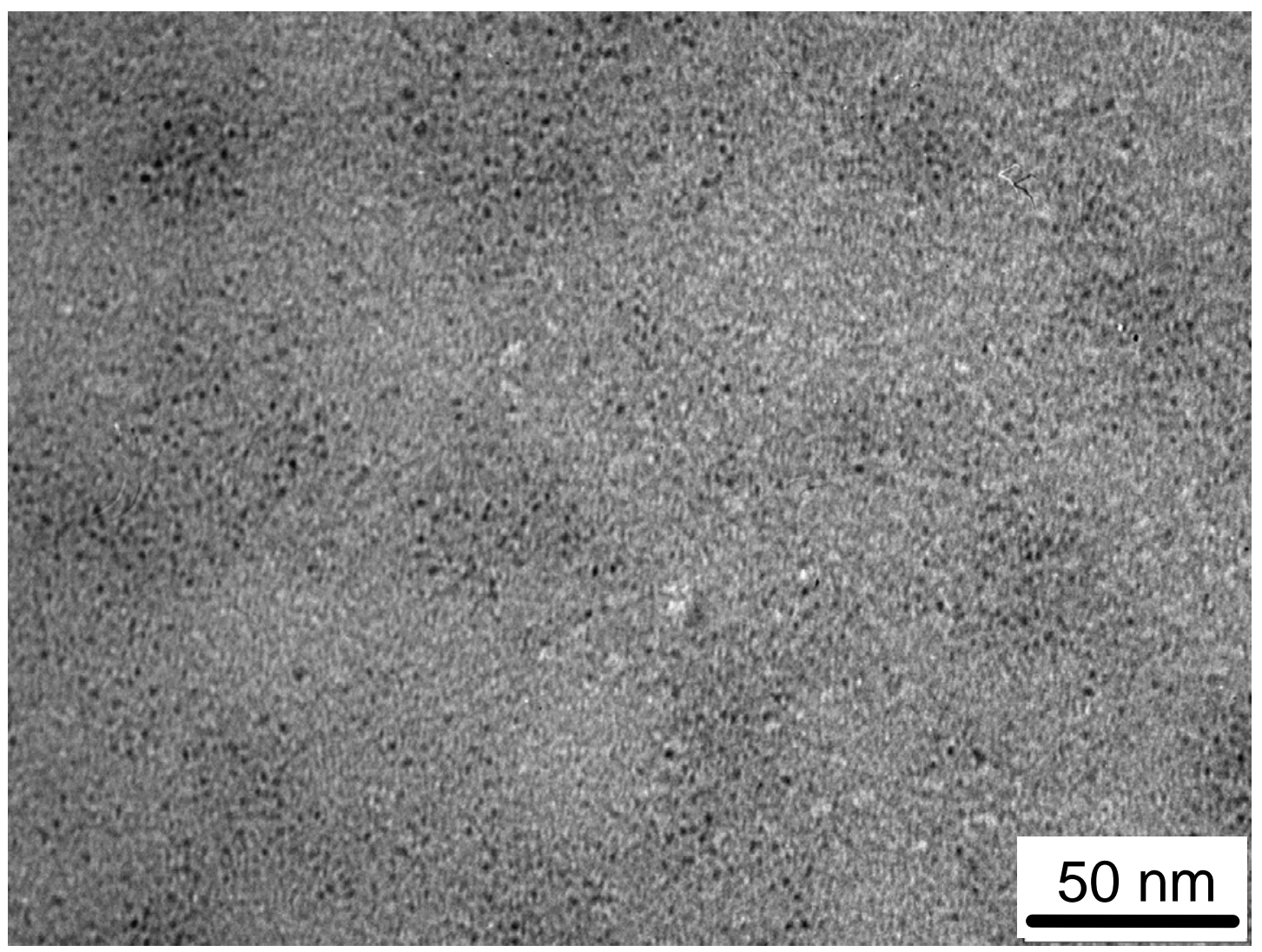

Figure S2. TEM image of a [PAA/PEI-Pd(0) $]_{3}$ PAA film on a carbon-coated copper grid. This image was taken at a lower magnification than Figure 1 in the article to better show the distribution of nanoparticles. 


\section{Calculation of TOFs from GC data with Response Factors}

\section{Determination of response factors:}

$\frac{\mathrm{S}_{\mathrm{P}}}{\mathrm{S}_{\mathrm{R}}}=\frac{\mathrm{C}_{\mathrm{P}}{ }^{*} \mathrm{k}_{1}}{\mathrm{C}_{\mathrm{R}}}$
$\frac{\mathrm{S}_{1}}{\mathrm{~S}_{\mathrm{R}}}=\frac{\mathrm{C}_{1}{ }^{*} \mathrm{k}_{2}}{\mathrm{C}_{\mathrm{R}}}$

Where $\mathrm{S}_{\mathrm{P}}=$ Peak area corresponding to product

$$
\begin{aligned}
& S_{I}=\text { Peak area corresponding to isomer } \\
& S_{R}=\text { Peak area corresponding to Reactant } \\
& C_{P}=\text { Concentration of product } \\
& C_{R}=\text { Concentration of reactant } \\
& C_{I}=\text { Concentration of isomer } \\
& k_{1}=\text { Response factor for product to reactant } \\
& k_{2}=\text { Response factor for isomer to reactant }
\end{aligned}
$$

Response factors were calculated from the signals due to a solution containing $10 \mathrm{mM}$ product, $10 \mathrm{mM}$ reactant, and $10 \mathrm{mM}$ isomer, and three chromatograms with three standard solutions were averaged to determine the value.

\section{Calculation of \% hydrogenation:}

$1 \mathrm{~mL}$ samples were removed every few minutes (time intervals depended on the rate) from the hydrogenation mixture and placed in autosampler vials for GC analysis. Percent hydrogenation was determined as shown below.

$$
\% \text { hydrogenation }=\frac{C_{P}}{C_{P}+C_{R}+C_{1}} \times 100 \%=\frac{S_{P}{ }^{*} k_{2}}{S_{P}{ }^{*} k_{2}+S_{R}{ }^{*} k_{1}{ }^{*} k_{2}+S_{1}{ }^{*} k_{1}} \times 100 \%
$$


When the rates of hydrogenation and isomerization were added,

$\%$ reaction $=\%$ of hydrogenation $+\%$ of isomerization:

$$
\% \text { reaction }=\frac{C_{P}+C_{I}}{C_{P}+C_{R}+C_{I}} \times 100 \%=\frac{S_{P}{ }^{*} k_{2}+S_{1}{ }^{*} k_{1}}{S_{P}{ }^{*} k_{2}+S_{R}{ }^{*} k_{1}{ }^{*} k_{2}+S_{1}{ }^{*} k_{1}} \times 100 \%
$$

Example data for the hydrogenation of Crotyl alcohol by [PAA/PEI-Pd(0) $]_{3} P A A$ in water.

Table S1. \%Hydrogenation of crotyl alcohol at different times.

\begin{tabular}{|c|c|}
\hline Time (min) & \% of hydrogenation \\
\hline 0.0 & 0 \\
\hline 10.0 & 7.9 \\
\hline 20.0 & 24.3 \\
\hline 30.0 & 33.9 \\
\hline 40.0 & 45.3 \\
\hline
\end{tabular}




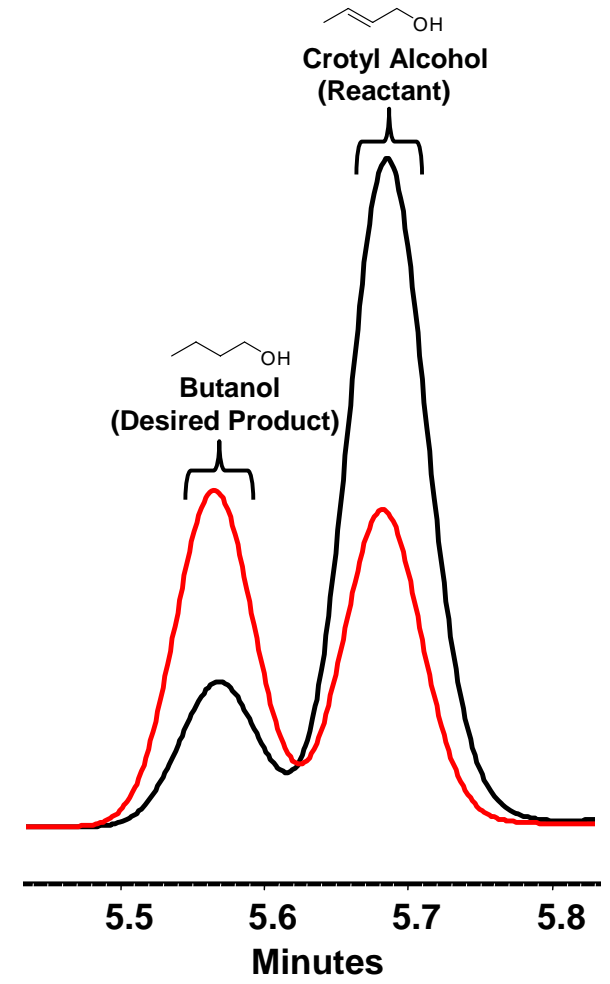

Figure S3: Example gas chromatograms of the reaction mixture from hydrogenation of crotyl alcohol (3) after 10 min (black line) and 40 min (red line) of reaction time. The reaction was catalyzed by $[\mathrm{PAA} / \mathrm{PEIPd}(0)]_{3} \mathrm{PAA}$ in water. Integration was performed using instrument software.

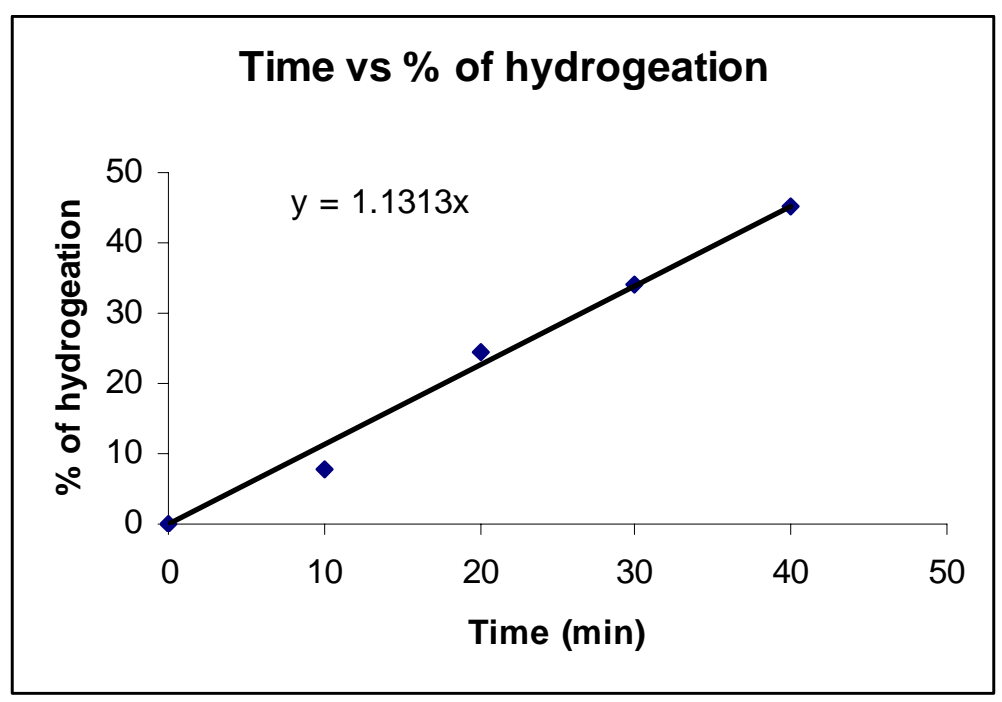

Figure S4: Plot of \% of hydrogenation vs time for hydrogenation of crotyl alcohol using $[\mathrm{PAA} / \mathrm{PEIPd}(0)]_{3} \mathrm{PAA}$ as a catalyst in water. 


\section{Final Calculation of TOF (moles hydrogenated/(moles Pd h):}

$$
\begin{aligned}
& 100 \% \text { hydrogenation }=1.25 \text { mmole } \\
& \text { TOF }=\frac{\text { slope }(\% \text { hydrogenation } / \mathrm{min})}{100 \%} * \frac{1.25 \times 10^{-3} \mathrm{moles}^{*} 60(\mathrm{~min} / \mathrm{h})}{3.19 \times 10^{-6} \mathrm{~mole} \mathrm{Pd}}
\end{aligned}
$$

The amount of Pd in the $250 \mathrm{mg}$ of [PAA/PEI-Pd(0)] $]_{3} \mathrm{PAA}$ on alumina catalyst used for the reaction was determined by atomic emission spectroscopy to be $3.19 \times 10^{-6}$ mole. For this specific example,

$$
\mathrm{TOF}=\frac{1.1313(\% \text { hydrogenation } / \mathrm{min})}{100 \%} * \frac{1.25 \times 10^{-3} \mathrm{moles}^{*} 60(\mathrm{~min} / \mathrm{h})}{3.19 \times 10^{-6} \mathrm{~mole} \mathrm{Pd}}=266 \frac{\text { moles hydrogenated }}{\text { moles Pd h }}
$$




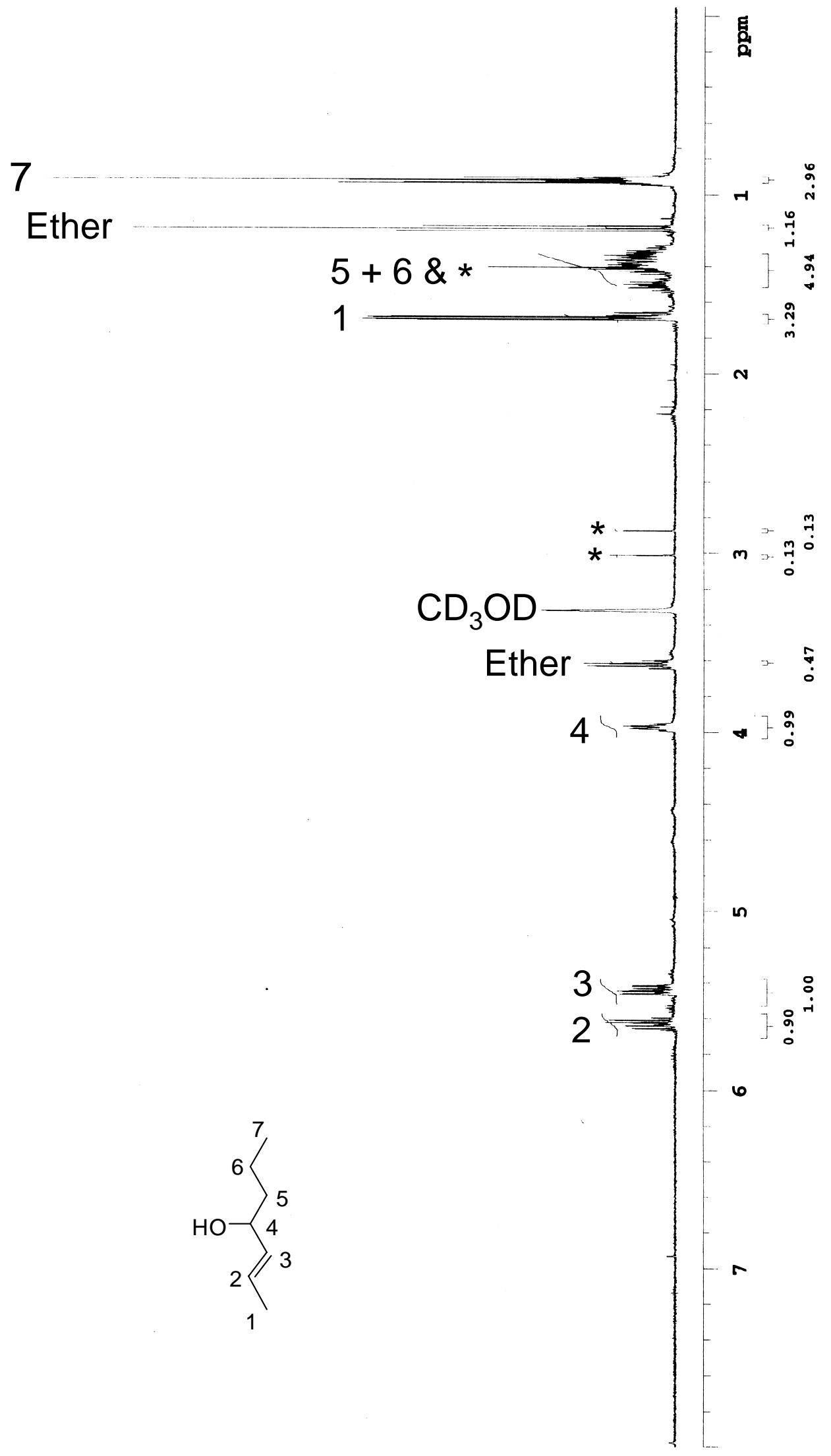

Figure S5. ${ }^{1} \mathrm{H}$ NMR spectrum of isolated crude compound 6 in $\mathrm{CD}_{3} \mathrm{OD}$. Impurity peaks are indicated with a * 


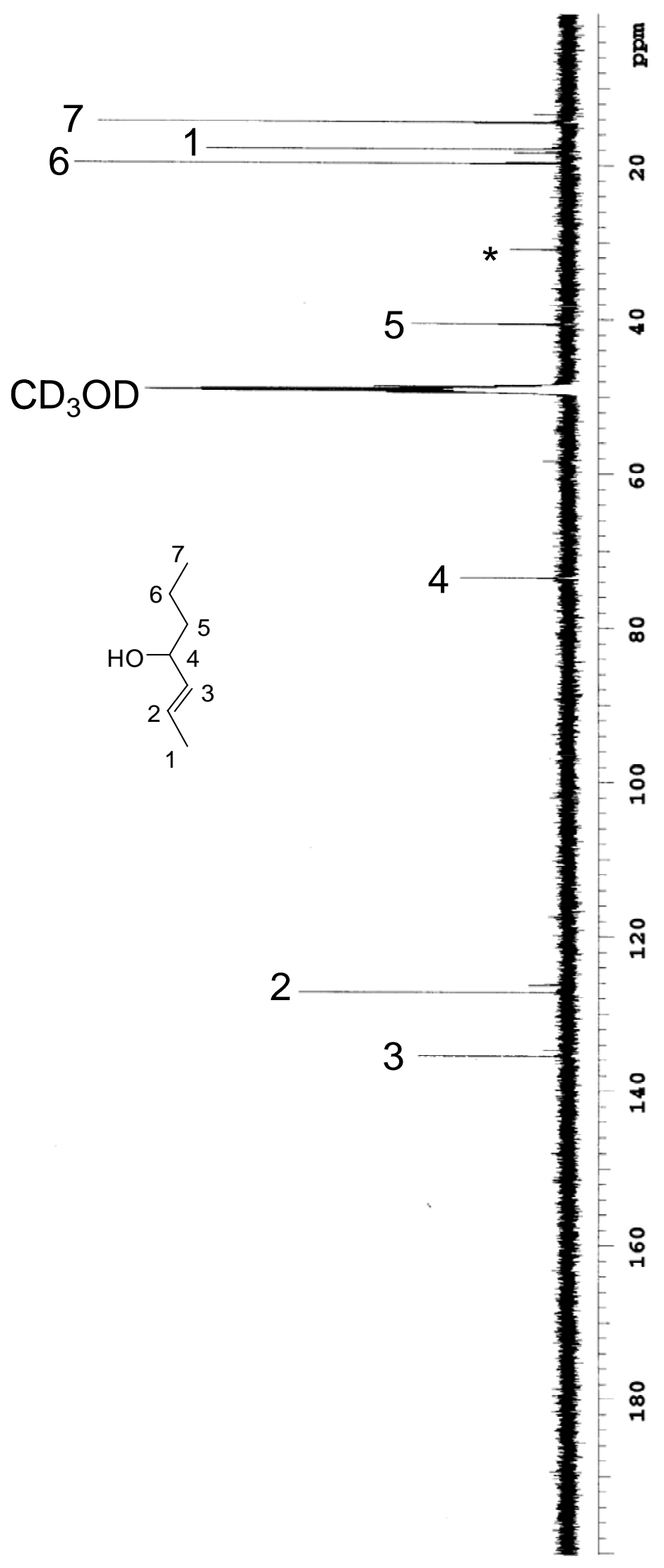

Figure S6. ${ }^{13} \mathrm{C}$ NMR spectrum of isolated crude compound 6 in $\mathrm{CD}_{3} \mathrm{OD}$. Impurity peaks are indicated with a *. 


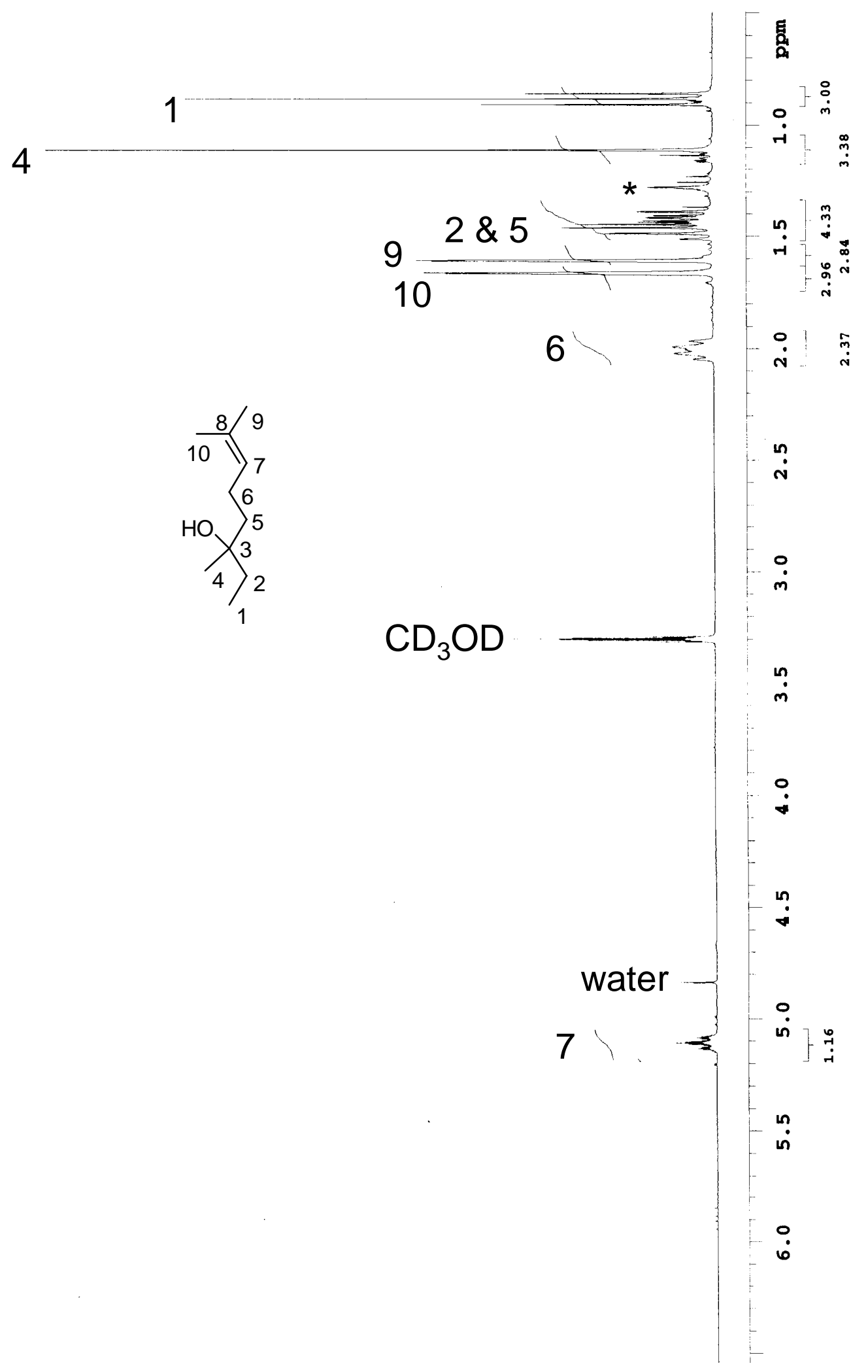

Figure S7. ${ }^{1} \mathrm{H}$ NMR spectrum of purified compound $\mathbf{8}$ in $\mathrm{CD}_{3} \mathrm{OD}$. Impurity peaks are indicated with a * 


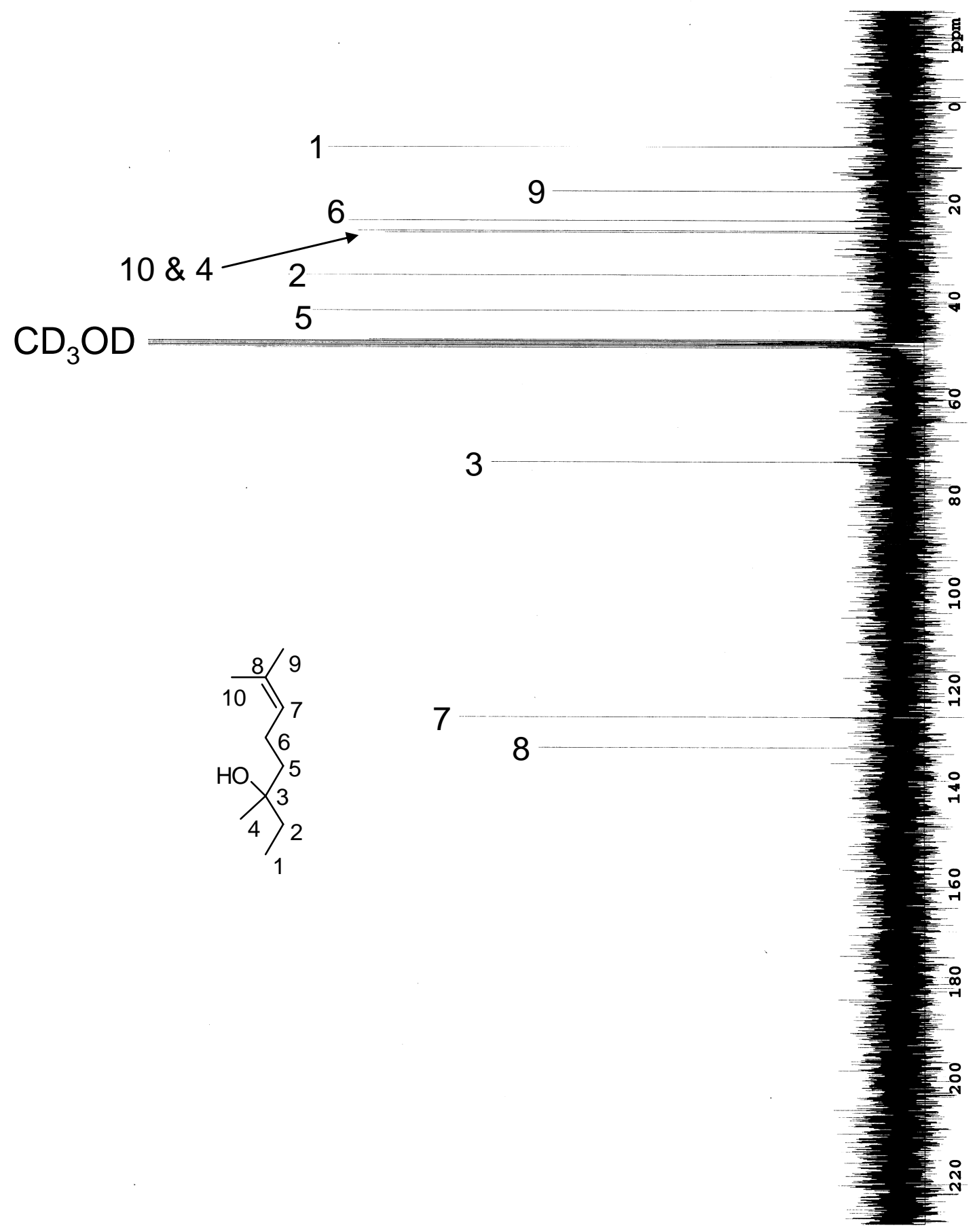

Figure S8. ${ }^{13} \mathrm{C}$ NMR spectrum of purified compound 8 in $\mathrm{CD}_{3} \mathrm{OD}$. 